\title{
Erratum a: Taddeo Alderotti e le radici medioevali della terapia steroidea
}

\section{Roberto Toni}

Pubblicato online: 22 ottobre 2014

(C) Springer International Publishing AG 2014

Erratum a: l'Endocrinologo (2014) 15: 135-138

DOI 10.1007/s40619-014-0033-x

Il testo a pag. 135 , riga 12 , riporta un riferimento errato al testo "Anothomia (1312)" il riferimento corretto è "Anothomia (1316)".

La versione online dell' articolo originale può essere trovata al doi:10.1007/s40619-014-0033-x.

R. Toni $(\varangle)$

Unità di Antropometria e Medicina delle Costituzioni, Centro Interdipartimentale di Medicina dello Sport e dell'Esercizio

Fisico, Università degli Studi di Parma, Parma, Italia

e-mail: roberto.toni@unipr.it

R. Toni

e-mail: roberto.toni@unibo.it

R. Toni

Department of Medicine, Division of Endocrinology, Diabetes and Metabolism, Tufts Medical Center-Tufts University School of Medicine, Boston, MA, USA

e-mail: roberto.toni@tufts.edu 